\title{
In reply: Comment on an article written by Vanita Ahuja et al.?
}

\author{
Vanita Ahuja $\cdot$ Sunita Kazal $\cdot$ Deepak Thapa
}

Received: 15 June 2014/ Accepted: 16 June 2014/Published online: 6 July 2014

(C) Japanese Society of Anesthesiologists 2014

Keywords Foreign body · Difficult intubation · Infant feeding tube

To the Editor:

We appreciate the authors for reading the article with great interest. The soft tissue neck X-ray of a 1-year-old infant with aerodigestive foreign body showed a radiopaque foreign body with subglottic airway compromise and a visibly normal airway below it. Following tracheal intubation with the 8-F infant feeding tube, the lungs were ventilated using the Mapleson F infant T-piece breathing system with hand ventilation. The initial $\mathrm{EtCO}_{2}$ was high $(45-55 \mathrm{mmHg})$ but then decreased gradually $(40-42 \mathrm{mmHg})$ with hand ventilation at 20-24 breaths/ min. We used hand ventilation to avoid loss of shared airway with the surgeon and a continuous feel of the resistance and compliance as any changes can influence it quickly. The surgeon was interrupted twice due to an increase in resistance to allow adequate lung ventilation. Following retrieval of the aerodigestive FB, the lungs were hyperventilated to achieve normocapnia. We agree that measurement of respiratory parameters within the circuit spirometry would have been useful with hand ventilation but this could have made the circuit heavy, leading to disconnections [1], and intraoperative benefits of its use in infants is inconclusive [2]. Post-procedure, the patient was managed in the postoperative care area with expertise and essential equipment to facilitate early airway management in the event of airway compromise.

Conflict of interest None.

\section{References}

1. Huffman LM. AANA journal course: new technologies in anesthesia: update for nurse anesthetists-monitoring ventilation and compliance with Side Stream Spirometry. AANA J. 1991; 59:249-59.

2. Manowska M, Bartkowska-Śniatkowska A, Kobylarz K, Piotrowski A, Walas W, Wołoszczuk-Gębicka B, Polish Society of Anaesthesiology and Intensive Therapy. The consensus statement of the paediatric section of the Polish Society of Anaesthesiology and Intensive Therapy on general anaesthesia in children under 3 years of age. Anaesthesiol Intensive Ther. 2013;45:119-33.

This author's reply refers to the letter to the editor at doi:10.1007/s00540-014-1881-z.

V. Ahuja $(\bowtie) \cdot$ S. Kazal · D. Thapa

Department of Anesthesia and Intensive Care, Government

Medical College and Hospital, Sector 32, Chandigarh, India

e-mail: vanitaanupam@yahoo.co.in 